\title{
Dietary Lipid:Protein Ratio and n-3 Long-Chain Polyunsaturated Fatty Acids Alters the Gut Microbiome of Atlantic Salmon Under Hypoxic and Normoxic Conditions
}

\author{
David Huyben ${ }^{1,2 *}$, Beeke K. Roehe ${ }^{1}$, Michaël Bekaert ${ }^{1}$, Bente Ruyter ${ }^{3}$ and Brett Glencross ${ }^{1}$ \\ ${ }^{1} /$ nstitute of Aquaculture, University of Stirling, Stirling, United Kingdom, ${ }^{2}$ Department of Animal Biosciences, University of \\ Guelph, Guelph, ON, Canada, ${ }^{3}$ Norwegian Institute of Food, Fisheries, and Aquaculture Research (Nofima), Tromsø, Norway
}

Researchers have adjusted dietary lipid:protein ratios and n-3 long-chain polyunsaturated fatty acids (LC-PUFA) to optimize the growth performance of Atlantic salmon. However, dietary impacts on the gut microbiome are lacking, especially under varying environmental conditions. To examine this response, post-smolt salmon (184 $\pm 5 \mathrm{~g})$ were fed diets with lipid:protein ratios considered low (180, $570 \mathrm{~g} / \mathrm{kg})$ and high $(230,460 \mathrm{~g} / \mathrm{kg})$ along with

OPEN ACCESS

Edited by:

Kwangcheol Casey Jeong, University of Florida, United States

Reviewed by:

Konstantinos Ar. Kormas, University of Thessaly, Greece

Si Hong Park,

Oregon State University, United States

${ }^{*}$ Correspondence:

David Huyben

huybend@uoguelph.ca

Specialty section: This article was submitted to

Food Microbiology,

a section of the journal

Frontiers in Microbiology

Received: 31 July 2020 Accepted: 08 December 2020 Published: 23 December 2020

Citation:

Huyben D, Roehe BK, Bekaert M, Ruyter B and Glencross B (2020) Dietary Lipid:Protein Ratio and n-3 Long-Chain Polyunsaturated Fatty Acids Alters the Gut Microbiome of Atlantic Salmon Under Hypoxic and Normoxic Conditions.

Front. Microbiol. 11:589898. doi: 10.3389/fmicb.2020.589898 low and high levels of n-3 LC-PUFA (7 or $14 \mathrm{~g} / \mathrm{kg}$ ) while fish were reared under low and high levels of dissolved oxygen (6.7 or $8.0 \mathrm{mg} / \mathrm{L}$ ). At day 0,35 and 116, digesta in the distal intestine were collected and analyzed for viable counts and 165 ribosomal RNA (rRNA) genes (V4 region) using Illumina MiSeq. The reduction in oxygen had negligible effects, except on viable plate counts of total bacteria and an initial effect on beta-diversity. In contrast, the high lipid $(\mathrm{HL})$ diets had an increased alpha-diversity (e.g., Shannon and Chao-1) at day 0 and day 35 whereas high n-3 diets suppressed these indices at day 116. Generally, a reduction in alpha-diversity was observed over time and an interaction between lipid:protein ratio $\times$ n-3 was found. Between diets, beta-diversity and phyla abundance were similar as both Proteobacteria (44\%) and Firmicutes (21\%) dominated. However, at the genus level Aliivibrio, Streptococcus, Weissella, and Lactobacillus, were associated with low lipid (LL) diets while the high lipid diets were associated with less abundant bacteria, e.g., Chromohalobacter. At day 116, the relative abundance of the Tenericutes phylum increased 10 -fold (36\%). Fish fed the high lipid diet with high n-3 had reduced alpha-diversity, lowest abundance of lactic acid bacteria, and highest abundance of Mycoplasma, which may indicate a less healthy gut microbiome. Phylogenetic Investigation of Communities by Reconstruction of Unobserved States (PICRUSt) analysis revealed that saturated and unsaturated fatty acid biosynthesis pathways were several folds higher in fish fed the high lipid diet, possibly to compensate for the lack of dietary n-3. In summary, our results show that the viable plate counts, alpha-diversity, betadiversity, and predictive function of gut bacteria in Atlantic salmon post-smolts are influenced by dietary lipid:protein ratio and n-3 LC-PUFA over several time points with little effect by dissolved oxygen.

Keywords: bacteria, hypoxia, intestinal microbiota, salmonids, predictive metagenomic function, 16S rRNA gene 


\section{HIGHLIGHTS}

- Dissolved oxygen level had little to no effect on the gut microbiome.

- Alpha-diversity was affected initially by lipid:protein ratio, but later by n-3 LC-PUFA level.

- High lipid diets had reduced abundance of lactic acid bacteria replaced by Mycoplasma.

- Fish fed low n-3 had more gut bacteria related to fatty acid biosynthesis pathways.

\section{INTRODUCTION}

The gut microbiota (e.g., bacteria and fungi) play a vital role in fermenting dietary carbohydrates and producing short chain fatty acids (SCFA), such as acetate, propionate, and butyrate, which can be utilized in energy metabolism and enhance gut health (Kihara and Sakata, 1997; Asaduzzaman et al., 2018). Abundance of different phyla in the gut (e.g., Firmicutes) has been found to increase lipid deposition in zebrafish (Danio rerio; Semova et al., 2012). It is important to better understand the interactions between diet, gut microbes and growth performance of Atlantic salmon (Salmo salar) because they are an economically important fish to the aquaculture industry. Only two studies have used next-generation sequencing to determine the influence of dietary lipids on the salmon gut microbiota (Zarkasi et al., 2016; Rudi et al., 2018). Feeding high lipid:protein diets was found to increase the diversity and abundance of lactic acid bacteria in the gut of Atlantic salmon (Zarkasi et al., 2016). However, energy levels were not equal between diets and the gut bacteria were not associated with any functional role, e.g., using a predictive metagenomic approach.

Researchers have adjusted dietary levels of lipids, protein, and n-3 long-chain polyunsaturated fatty acids (LC-PUFA), such as eicosapentaenoic acid (EPA) and docosahexaenoic acid (DHA), to optimize the growth performance of Atlantic salmon (Salmo salar; Ruyter et al., 2000; Glencross et al., 2014; Bou et al., 2017). However, only a few studies have investigated the effects of lipid and n-3 LC-PUFA on the gut microbiome of salmon, which mainly look at replacing fish oil with vegetable oils as a sustainable alternative (Ringø et al., 2018). Feeding oils with different levels of $n-3$ and $n-6$ PUFA were found to influence viable counts of both lactic acid and pathogenic bacteria in the gut of Arctic charr (Salvelinus alpinus; Ringø, 1993; Ringø et al., 2002). However, replacing fish oil with vegetable oils was found to have minor changes on the gut microbiota of rainbow trout fry (Oncorhynchus mykiss; Ingerslev et al., 2014) and Atlantic salmon pre- and postsmolts (Rudi et al., 2018) using 16S rRNA gene next-generation sequencing. However, effects on gut microbiota may have been masked due to short experiment duration or fish life-stage changes. In addition, reductions in dissolved oxygen (i.e. hypoxia) have been found to change gut microbiota in temperate fish and shrimp (Fan et al., 2020; Sun et al., 2020). Hypoxia is a common stressor in salmon farm operations known to reduce feed intake (Huyben et al., 2021), although research on the hypoxic effects on gut microbial populations is lacking.
The objective of this study was to characterize the gut microbiota of Atlantic salmon at three different time points and determine if the viable plate counts, alpha-diversity, betadiversity, and predictive function of gut microbes can be influenced by the dietary lipid:protein ratio, level of $n-3$ PUFA, and dissolved oxygen. We hypothesized that high levels of n-3 PUFA and hypoxia influence the growth of certain gut microbes and reduce the alpha-diversity based on reduced reliance on fatty acid producing bacteria and reduced feed intake.

\section{MATERIALS AND METHODS}

\section{Fish and Facilities}

In May 2018, Atlantic salmon post-smolts were acquired from a local producer and reared at the Marine Environmental Research Laboratory (Machrihanish, United Kingdom). Fish were randomly allocated to 24 circular tanks containing 40 fish each. Each $500 \mathrm{~L}$ tank was equipped with a LED light, air stone, and automated feeder (Arvo-tec Oy, Huutokoski, Finland). Fish $(184.4 \pm 4.9$ g; mean \pm SD) were acclimated on a commercial diet (3 mm Intro, Biomar Ltd., Grangemouth, United Kingdom) for 3 weeks before the start of the experiment. During the 116 day (17 week) experiment, fish were fed twice per day for $3 \mathrm{~h}$ durations and the uneaten feed waste was collected each morning to allow for estimation of daily feed intakes in each tank. The flow rate and aeration were reduced in half of the tanks to apply a hypoxic stressor. The low oxygen tanks had a dissolved oxygen concentration of $78.0 \pm 2.3 \%$ $(6.7 \pm 0.2 \mathrm{mg} / \mathrm{L})$ compared to an oxygen saturation of $92.6 \pm 2.7 \%$ $(8.0 \pm 0.2 \mathrm{mg} / \mathrm{L})$ for the high oxygen tanks. The filtered seawater had a temperature of $13.2 \pm 0.2^{\circ} \mathrm{C}$ (mean $\pm \mathrm{SD}$ ). This experiment was approved by the Animal Welfare and Ethical Review Body (reference AWERB/1617/84) in accordance with the United Kingdom Home Office under the Animals (Scientific Procedures) Act 1986.

Groups of fish were fed one of four diets that were formulated to have markedly different lipid:protein ratios $(26.2 \mathrm{~g} / \mathrm{MJ}$ or $21.0 \mathrm{~g} / \mathrm{MJ})$ based on combinations of high protein $(570 \mathrm{~g} / \mathrm{kg})$ and low lipid (LL; $180 \mathrm{~g} / \mathrm{kg}$ ), or low protein $(460 \mathrm{~g} / \mathrm{kg}$ ) and high lipid (HL; $230 \mathrm{~g} / \mathrm{kg}$ ), but were all formulated to be isoenergetic on a digestible basis $(21 \mathrm{MJ} \mathrm{DE} / \mathrm{kg})$. Additionally, each set of diets had secondary treatments that varied in their level of n-3 PUFA (7 or $14 \mathrm{~g} / \mathrm{kg}$ ). The $3 \mathrm{~mm}$ pellets were produced using extrusion processing by a commercial manufacturer (Sparos I\&D, Olhão, Portugal).

\section{Sample Collection}

Fish at day 21 (fed commercial diet), 0 (initial feeding of test diets), 35 (short-term feeding), and 116 (long-term feeding) were overdosed with tricaine methanesulfonate (MS-222), cervical vertebrae were dislocated, and their abdomen was dissected. A sterile scalpel blade was used to cut the distal intestine at the ileo-rectal valve and $0.5 \mathrm{~cm}$ before the anus. Using sterile forceps, the fecal content (hindgut digesta) was firmly squeezed into a sterile cryotube in order to collect both autochthonous 
and allochthonous bacteria, frozen on dry ice, and stored at $-70^{\circ} \mathrm{C}$. Nine fish were randomly sampled at day $21(N=9)$, three from each diet at day $0(N=27)$, and nine from each diet at day $35(N=72)$ and $116(N=72)$. In addition, triplicate samples of each experimental diet and the commercial diet were collected from newly opened bags, homogenized, frozen, and stored at $-70^{\circ} \mathrm{C}$. At day 116 , the tanks were drained and a sterile cotton swab was used to collect biofilm from the side of three randomly chosen tanks to act as an environmental sample, which was frozen and stored at $-70^{\circ} \mathrm{C}$.

\section{Viable Plate Counts of Bacteria}

Plate counts of viable bacteria were performed on two different media of either typtone soya agar (TSA; Sigma-Aldrich, Dorset, United Kingdom) or Man-Rogosa-Sharpe agar (MRS; SigmaAldrich) to isolate total and lactic acid bacteria on days 21, 0,35 , and 116 . First, approximately $100 \mathrm{mg}$ of feces was added to $900 \mu \mathrm{l}$ sterile $0.85 \% \mathrm{NaCl}$, vortexed, and serial diluted. Four droplets of $10 \mu \mathrm{l}$ each were pipetted onto agar plates for each dilution, dried at room temperature for $2 \mathrm{~h}$, sealed with parafilm, and then incubated at $22^{\circ} \mathrm{C}$ for $4-6$ days. Mean counts per droplet were divided by the dilution factor of both the droplet volume and dilution series to calculate total viable counts as colony forming units (CFUs) per $\mathrm{ml}^{-1}$. Some viable plate count samples were removed due to contamination or overgrowth of bacteria and fungi.

\section{$16 S$ rRNA Gene Extraction, Amplification, and Sequencing of Bacteria}

Processing and sequencing of bacteria were performed on two separate occasions; (1) day 0 and 35 gut samples and (2) day 116 gut and environmental samples. Day 21 samples were not included since their composition would be similar to day 0 . Approximately $150 \mathrm{mg}$ of feces, diet, and tank biofilm swab were each homogenized in $1 \mathrm{ml}$ of Inhibit EX buffer (Qiagen Ltd., Manchester, United Kingdom) using a Mini-bead-breaker 16 (Biospec Products Inc., Bartlesville, OK, United States) for four cycles of $30 \mathrm{~s}$ with $60 \mathrm{~s}$ rest. The DNA was extracted using QIAamp Fast DNA Stool Mini kit (Qiagen Ltd.) according to the manufacturer's instructions. In addition, samples were incubated for $10 \mathrm{~min}$ at $95^{\circ} \mathrm{C}$ to reduce foam formation after homogenization and to enhance lysis of Gram-positive bacteria. Two samples of nuclease free water were extracted, amplified, and sequenced to represent negative controls and to identify potential contaminants. Samples were eluted with $20 \mu \mathrm{l}$ of nuclease free water into new tubes. The nucleic acid and DNA concentrations were quantified using a ND-1000 Nanodrop spectrophotometer (Nanodrop Technologies LLC, Wilmington, DE, United States) and a Quibit 2.0 fluorimeter (Invitrogen, Thermo Fisher Scientific, Hempstead, United Kingdom). Samples were diluted to a concentration of $25 \mathrm{ng} / \mathrm{ml}$ of nucleic acid (Nanodrop) or $2.5 \mathrm{ng} / \mathrm{ml}$ of DNA (Quibit).

A PCR was performed on $25 \mu \mathrm{l}$ reactions consisting of $2 \mu \mathrm{l}$ template (5 ng of DNA), $1.25 \mu \mathrm{l}(10 \mu \mathrm{M})$ of each forward primer (515F; GTGYCAGCMGCCGCGGTAA) and reverse primer (806R; GGACTACNVGGGTWTCTAAT; Caporaso et al., 2011) with Illumina adapter to target the V4 region of the 16S rRNA gene, nuclease free water, and $12.5 \mu$ l of $2 x$ NEBNext Ultra II Q5 Master Mix (New England Biolabs Ltd., Hitchin, United Kingdom). The PCR was performed in a T-advanced thermocycler (Biometra GmbH, Göttingen, Germany) under condition: $98^{\circ} \mathrm{C}$ for $60 \mathrm{~s}$ followed by 30 cycles of $98^{\circ} \mathrm{C}$ for $10 \mathrm{~s}, 53^{\circ} \mathrm{C}$ for $10 \mathrm{~s}$, and $65^{\circ} \mathrm{C}$ for $45 \mathrm{~s}$ with a final step of $65^{\circ} \mathrm{C}$ for $5 \mathrm{~min}$. Amplicons were confirmed on a $1 \%$ agarose gel alongside negative controls of nuclease free water instead of template. Samples were purified with Axygen AxyPrep Mag PCR clean up kit (Corning Inc., Corning, NY, United States) using magnetic beads at a ratio of $1: 1$ and two washes of $70 \%$ $\mathrm{EtOH}$, according to the manufacturer's instructions. Samples were eluted with $20 \mu \mathrm{l}$ of $10 \mathrm{mM}$ Tris (Qiagen Ltd.) and $7.5 \mu \mathrm{l}$ was used in a second PCR with the above conditions, except only for 10 cycles and the forward and reverse primers consisted of different combinations of eight basepairs from the Nextera XT DNA Library Preparation kit (Illumina Inc., Cambridge, United Kingdom) to individually index each sample. Samples were purified with Axygen magnetic beads (Corning Inc.) as above, quantified with a Quibit 2.0 fluorimeter (Thermo Fisher Scientific), and diluted with $10 \mathrm{mM}$ Tris (Qiagen Ltd.) to a concentration of $2.81 \mathrm{ng} / \mu \mathrm{l}$ or $10 \mathrm{nM}$ based on a final PCR product of 428 basepairs and a molar weight of $656.6 \mathrm{M}$. An equal volume of each sample was pooled, confirmed on a $1 \%$ agarose gel, and sequenced on the Illumina MiSeq platform at the University of Stirling (Stirling, United Kingdom) with a MiSeq Reagent kit v2 of 500 cycles (Illumina Inc.).

\section{Bioinformatics}

The 16S rRNA gene sequences were analyzed using Mothur version 1.42.3 (Schloss et al., 2009) according to the MiSeq SOP (https://www.mothur.org/wiki/MiSeq_SOP; Kozich et al., 2013). Sequence reads which were smaller than $200 \mathrm{bp}$, larger than $300 \mathrm{bp}$, had more than eight consecutive bp and were outside the V4 region of the 16S rRNA gene were removed from the dataset. Filtered sequence reads were aligned to the SILVA reference database version 123 (Quast et al., 2012), pre-clustered to merge sequences with less than 2 bp difference, and chimeras were removed using the open-source tool VSEARCH (Rognes et al., 2016). Sequences were classified using the RDP Bayesian Classifier trainset version 16.0 at a cut off of $80 \%$ (Cole et al., 2013), and taxon resembling chloroplasts, mitochondria, unknowns, archaea, and eukaryotes were removed. Count tables of unique sequences were created to reduce processing time. Sequences were clustered and classified into operational taxonomic units (OTUs) at a cut off of 0.03. Ralstonia (genus level) was identified as a contaminant based on previous research on common laboratory contaminants in microbiome studies (Salter et al., 2014) and its high abundance of 84 and $53 \%$ on days $0 / 35$ and 116 in the blank samples, thus Ralstonia was removed from both datasets. Sequences were subsampled in order to normalize all samples to the lowest number of sequences per samples, which was 8,257 and 8,072 for day $0 / 35$ and 116 datasets, respectively. The raw $16 \mathrm{~S}$ rRNA gene sequence reads were deposited in the Sequence 
Read Archive of NCBI and made publicly available under BioProject Accession number PRJNA650141. ${ }^{1}$

\section{Statistical Analysis}

Normal distribution and homogeneity of each dataset were determined using Shapiro-Wilk and Levene tests in $\mathrm{R}$ version (R-Core-Team, 2015). When needed, data were normalized by log, square-root, or arcsine transformation. All data are presented as means \pm SE unless otherwise specified. Akaike Information Criterion (AIC) was used to determine the statistical model that best fit the data. Differences between treatments were determined using linear mixed effects $(l m e)$ or linear model $(l m)$ based on the nlme and stats packages in R v3.5 (Pinheiro et al., 2014). In lme model, fixed effects were lipids (lipid:protein ratio), n-3 PUFA and oxygen with fish tank as a random effect and interactions between all three factors ( $y=$ lipids $\times \mathrm{n} 3 \times$ oxygen +1 tank). In $l m$ model, fixed effects and interactions were the same $(y=$ lipids $\times \mathrm{n} 3 \times$ oxygen $) . p$-values from the models were generated using ANOVA tables and differences between treatments were determined using lsmeans. $p$-values below 0.05 were considered significant and $p$-values between 0.05 and 0.10 were considered to be a tendency.

For alpha-diversity, rarefaction.single, and summary.single commands in Mothur were used on subsampled OTU datasets to generate rarefaction curves and diversity tables based on Good's coverage, observed species, Shannon diversity (non-parametric), and Chao-1 richness indices. These indices were analyzed to measure the total species represented, abundance/evenness of species, and the richness of rare species in the gut community. Significant differences between treatments were determined using $l m e$ and $l m$ models in $\mathrm{R}$ as mentioned above. For beta-diversity, Bray-Curtis distance matrixes were square-root transformed and significance of each factor (e.g., lipid, n-3 PUFA, and oxygen) was determined using Analysis of Similarity (ANOSIM) with the adonis function based on the vegan package in $\mathrm{R}$ (Oksanen et al., 2018). Beta diversity was plotted using 2D Non-metric Multidimensional Scaling (NMDS). Linear discriminant analysis (LDA) Effect Size (LefSe) was used to identify OTUs that explain differences between treatments using Kruskal-Wallis tests and a LDA threshold of 2.0 (Segata et al., 2011).

\section{Predictive Metagenomic Analysis}

In Mothur, the SILVA aligned and chimera-removed fasta file from the previous section was classified using the Greengenes reference database version gg_13_8_99 (Desantis et al., 2006), and non-bacteria were removed and the distance matrix was clustered as above. In order to use Phylogenetic Investigation of Communities by Reconstruction of Unobserved States (PICRUSt), an OTU table was generated using make.biom with Greengenes taxonomy and PICRUSt OTU map. In VirtualBox (Oracle, Redwood Shores, CA, United States), Microbiome Helper version 0.4 was used to normalize the OTU copy number across samples, make functional predictions using

${ }^{1}$ https://www.ncbi.nlm.nih.gov/bioproject/PRJNA650141
Kyoto Encyclopedia of Genes and Genomes (KEGG) database (Kanehisa and Goto, 2000) and collapse KEGG Orthologs into KEGG pathway predictions according to the PICRUSt tutorial (Langille et al., 2013). PICRUSt predictions were visualized using Statistical Analysis of Taxonomic and Functional Profiles (STAMP) software version 2.1.3 (Parks et al., 2014) by comparing each pair of treatments within each factor $(N=72)$ using extended error bar plots at three KEGG pathway levels. Significant differences for predictive metagenomic pathways were determined using Welch's two-sided $t$-test (unequal variance) and a Bonferroni correction was applied to $p$-values to account for multiple testing.

\section{RESULTS}

\section{Growth Performance of Atlantic Salmon}

Final weight was significantly affected by lipid:protein ratio $(p=0.031)$, $\mathrm{n}-3(p=0.007)$, and oxygen $(p<0.001)$ while feed intake was only affected by oxygen $(p=0.001)$. Final weight was highest for fish fed the high lipid diet with high n-3 LC-PUFA under high oxygen $(552 \pm 4 \mathrm{~g} /$ fish, mean $\pm \mathrm{SE})$ with a feed intake of $318 \pm 7 \mathrm{~g} / \mathrm{fish}$. The lowest final weight was for fish fed the low lipid diet with low n-3 under low oxygen at $436 \pm 17 \mathrm{~g} /$ fish with a feed intake of $210 \pm 16 \mathrm{~g} / \mathrm{fish}$. Further details on the phenomic responses of the trial are published by Huyben et al. (2021).

\section{Viable Plate Counts of Bacteria}

Total viable counts of aerobic bacteria cultured on TSA significantly decreased $(p=0.029)$ by approximately 3 -logs over the course of the experiment from $3.7 \pm 2.2 \times 10^{6}(6.6 \log )$ $\mathrm{CFU} \mathrm{ml} l^{-1}$ at day 21 compared to $4.2 \pm 1.0 \times 10^{3}(3.6 \mathrm{log})$ $\mathrm{CFU} \mathrm{ml} \mathrm{m}^{-1}$ at day 116 (Figure 1A). No significant effects of lipid, $\mathrm{n}-3$, or oxygen were found $(p>0.05)$, except for a significant lipid $\times$ oxygen interaction at day $116(p=0.032)$. Fish fed low lipid and kept at high oxygen had higher bacterial counts in their distal intestine.

Total viable counts of lactic acid bacteria cultured on MRS remained the same $(p=0.867)$ over the experiment with a mean count on day 116 of $2.9 \pm 0.7 \times 10^{3}(3.5 \log )$ CFU $\mathrm{ml}^{-1}$ (Figure 1B). No significant effects of lipid, n-3, oxygen, or interactions between the three factors were found $(p>0.05)$.

\section{Alpha-Diversity of 16S rRNA Gene Sequences}

There was no difference in Shannon diversity or Chao-1 richness between fish gut bacteria sampled at day 0 and $35(p=0.155$ and 0.682 ). On day 35, Good's coverage, number of OTUs, Shannon diversity, and Chao-1 richness were all significantly affected by lipid level in the diet while no effects of n-3 or oxygen were found (Table 1). In general, alpha-diversity increased when fish were fed high lipid diets and the lowest diversity was found for fish fed low lipid diets at high oxygen. Fish fed the high lipid diet with low n-3 had the highest Shannon diversity at low oxygen and highest Chao-1 richness at high oxygen. All indices were similar between day 0 and 35 
A

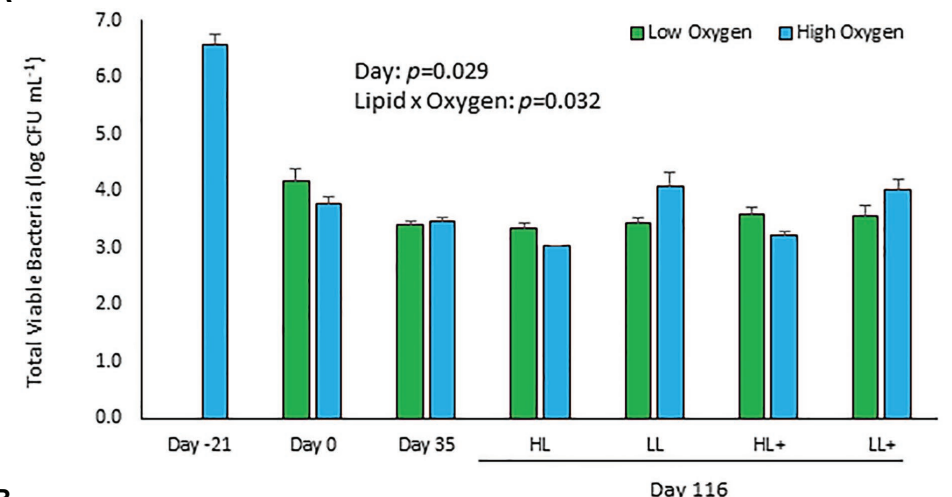

B

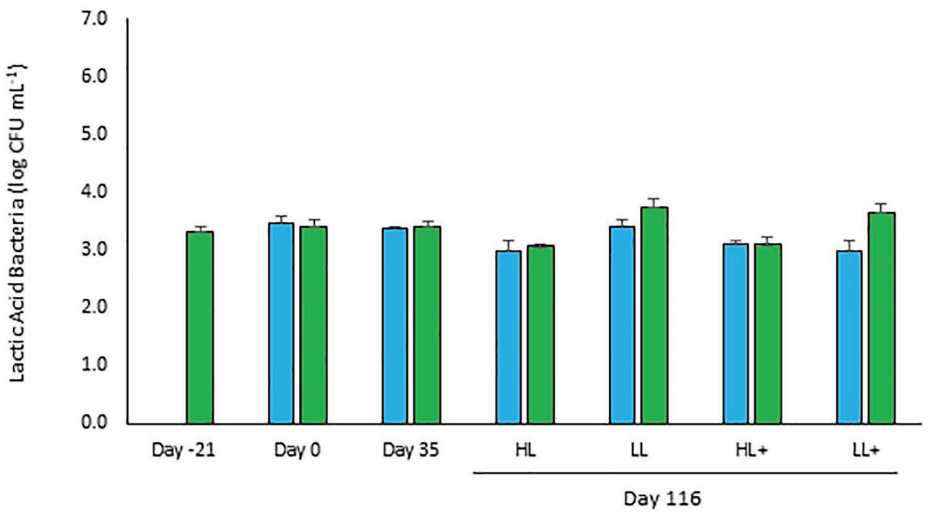

FIGURE 1 | Viable plate counts of (A) total and (B) lactic acid bacteria ( \pm SE) cultured on typtone soya agar (TSA) and man-rogosa-sharpe agar (MRS) plates of feces collected from the distal intestine of Atlantic salmon fed $\mathrm{HL}$ or LL diets with or without additional n-3 PUFA (+) and reared at low or high oxygen. Fish were sampled at $-21(N=9), 0(N=23), 35(N=48)$, and 116 days $(N=55)$. Some samples excluded due to fungal overgrowth.

TABLE 1 | Alpha-diversity indices of intestinal bacteria from Atlantic salmon fed high lipid (HL) or low lipid (LL) with or without additional n-3 polyunsaturated fatty acids [PUFA (+)] for $0(N=9)$ and 35 days $(N=72)$ and reared at low and high oxygen.

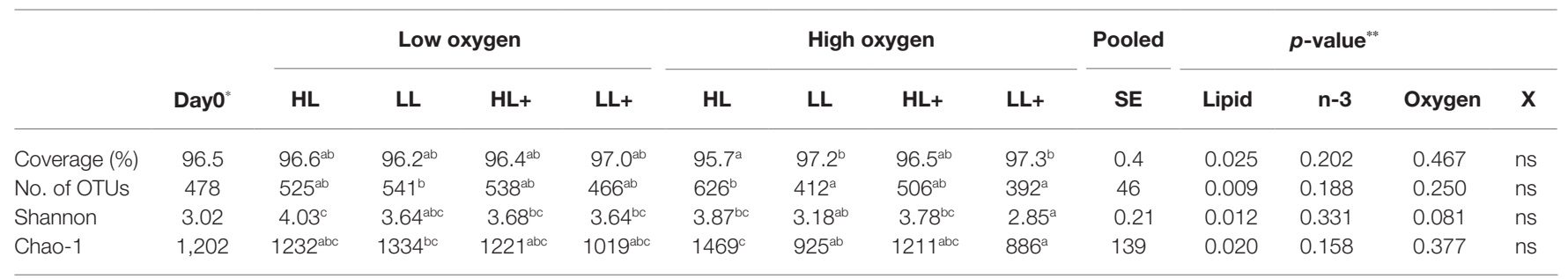

"all indices were similar between days 0 and 35 ( $p>0.05)$, but both were significantly different $(p<0.05)$ from gut bacteria at day $116($ Table 2$) .{ }^{* *} p$-values from Ime model with lipid, $n-3$ and oxygen as fixed effects, interactions between the three and fish tank as a random effect. Differing lowercase letters indicate significant difference ( $p<0.05)$.

$(p>0.100)$, but both were significantly different $(p<0.05)$ compared to those on day 116 as a clear reduction in Chao-1 richness was found over time (Figure 2).

On day 116, Good's coverage, number of OTUs, Shannon diversity, and Chao-1 Richness were all significantly affected by $n-3$ level in the diet while no effects of lipid or oxygen were found (Table 2). In general, alpha-diversity increased when fish were fed low n-3 diets. At high oxygen, fish fed the high lipid diet with low n-3 had the highest diversity and fish fed the high lipid diet with high n-3 had the lowest diversity, which was supported by a significant lipid $\times$ n-3 interaction for Shannon diversity. Regarding the environmental samples, Shannon and Chao-1 were different between the gut and tank biofilm $(p<0.001)$ and between the diet and tank biofilm ( $p=0.001)$, although no differences existed between the gut and diet $(p>0.05)$. Number of OTUs was more than 10-fold higher in the tank biofilm compared to gut and diet samples.

\section{Beta-Diversity of 165 rRNA Gene Sequences}

Between day 0 and 35 there was an effect of day (time) on beta-diversity of gut bacteria $(p=0.001)$, as shown clearly by 


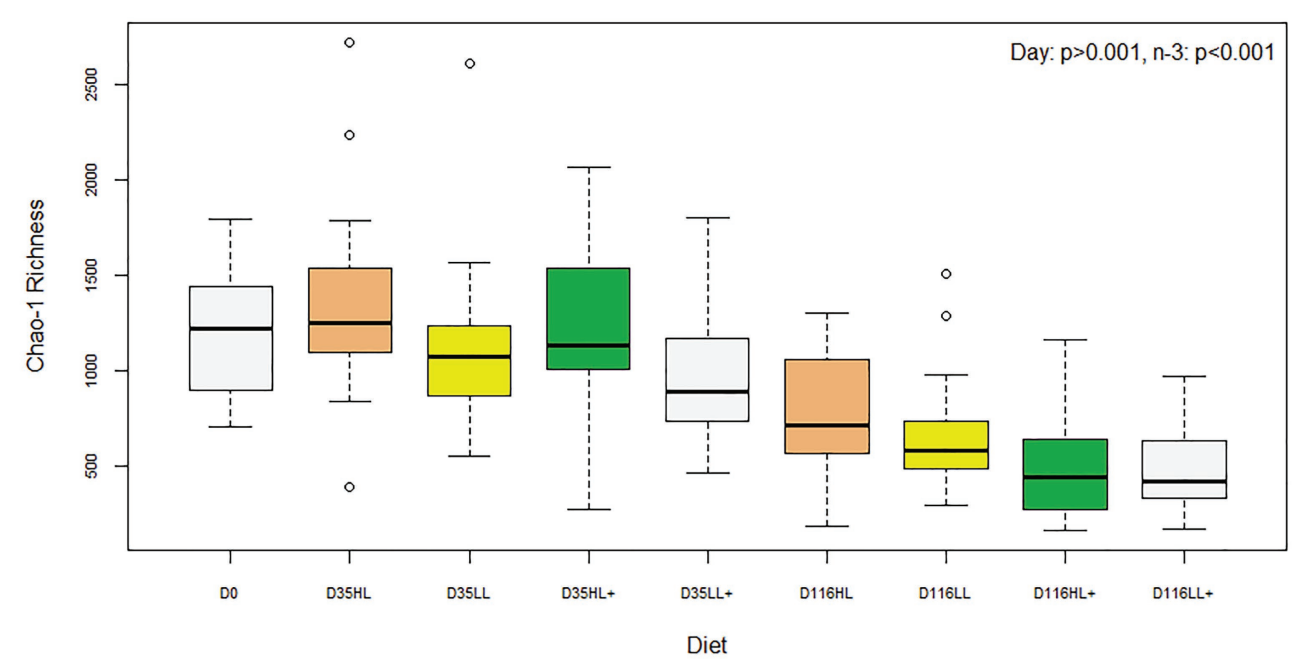

FIGURE 2 | Box plots of bacterial alpha-diversity from the distal intestine of Atlantic salmon fed HL or LL diets with or without additional n-3 PUFA (+). Oxygen conditions were pooled together and fish were sampled on day $0(N=9)$, day $35(N=72)$, and day $116(N=72)$.

TABLE 2 | Alpha-diversity indices of diet, tank biofilm ( $N=3)$, and intestinal bacteria from Atlantic salmon $(N=72)$ fed HL or LL with or without additional n-3 PUFA (+) for 116 days and reared at low and high oxygen.

\begin{tabular}{|c|c|c|c|c|c|c|c|c|c|c|c|c|c|c|c|}
\hline & \multirow[b]{2}{*}{ Diet } & \multirow[b]{2}{*}{ Tank } & \multicolumn{4}{|c|}{ Low oxygen } & \multicolumn{4}{|c|}{ High oxygen } & \multirow{2}{*}{$\frac{\text { Pooled }}{\text { SE }}$} & \multicolumn{3}{|c|}{$\begin{array}{c}p \text { - } \\
\text { value }^{* * *}\end{array}$} & \multirow[b]{2}{*}{$\mathbf{x}$} \\
\hline & & & HL & LL & HL+ & LL+ & $\mathrm{HL}$ & LL & HL+ & LL+ & & Lipid & $n-3$ & Oxygen & \\
\hline Coverage (\%) & 96.3 & 66.1 & $98.0^{\mathrm{bc}}$ & $98.1^{b c}$ & $98.2^{\mathrm{bc}}$ & $98.5^{c}$ & $96.6^{a}$ & $97.2^{\mathrm{ab}}$ & $98.6^{c}$ & $98.2^{\mathrm{bc}}$ & 0.4 & 0.645 & $<0.001$ & 0.068 & $n-3 \times 0$ \\
\hline No. of OTUs & 584 & 3,599 & $370^{b}$ & $330^{\mathrm{b}}$ & $324^{b}$ & $284^{a}$ & $562^{c}$ & $412^{\mathrm{bc}}$ & $234^{\mathrm{a}}$ & $349^{b}$ & 52 & 0.960 & 0.001 & 0.250 & ns \\
\hline Shannon & 3.62 & 7.60 & $2.86^{\mathrm{bc}}$ & $2.47^{b}$ & $2.38^{a b}$ & $2.64^{b}$ & $3.63^{c}$ & $2.43^{b}$ & $1.67^{\mathrm{a}}$ & $2.94^{b c}$ & 0.24 & 0.765 & 0.040 & 0.857 & $L \times n-3$ \\
\hline Chao-1 & 994 & 11,548 & $594^{a b}$ & $540^{\mathrm{ab}}$ & $530^{a b}$ & $445^{a}$ & $961^{c}$ & $795^{\mathrm{bc}}$ & $425^{a}$ & $532^{a}$ & 135 & 0.818 & $<0.001$ & 0.061 & ns \\
\hline
\end{tabular}

L, lipid; O, oxygen. ${ }^{* *} p$-values from Ime model with lipid, $n$-3 and oxygen as fixed effects, interactions between the three and fish tank as a random effect. Differing lowercase letters indicate significant difference $(p<0.05)$.

the separation in the NMDS plot (Figure 3A). On day 35, levels of lipid $(p=0.001)$ and oxygen $(p=0.005)$ affected beta-diversity of gut bacteria, but no effect of $n-3$ was found $(p=0.115)$ based on ANOSIM $(d f=68)$. On day 116 , differences in sample type (i.e., gut, diet, and tank biofilm) existed $(p=0.001)$ as clearly shown in the NMDS plot (Figure 3B). Levels of lipid $(p=0.009), \mathrm{n}-3(p=0.036)$, and a lipid $\times \mathrm{n}-3$ interaction $(p=0.001)$ affected beta-diversity of gut bacteria, but no effect of oxygen was found ( $p=0.367)$ based on ANOSIM $(d f=71)$.

Mean relative abundance at the phylum level on day 0 and 35 (Figure 4A) were dominated by Proteobacteria (44\%) followed by Firmicutes (21\%), Actinobacteria (4\%), and Tenericutes (3\%). On day 116 , gut bacteria were dominated by Tenericutes (36\%) followed by Firmicutes (24\%), Proteobacteria (11\%), and Actinobacteria (1\%; Figure 4B). Majority of bacteria in the diet were Firmicutes while the tank biofilm consisted of Proteobacteria and Bacteroidetes.

At the genus level, gut bacteria were dominated by Aliivibrio (22\%) followed by Streptococcus (7\%), Weissella (6\%), Rhodobacteraceae (4\%), Lactobacillus (4\%), and Mycoplasmataceae (3\%) on day 0 and 35 (Figure 5A). On day 116, gut bacteria were dominated by Mycoplasmataceae (32\%), Streptococcus (9\%), Weissella (9\%), Lactobacillus (4\%), Aliivibrio (4\%), Mycoplasma (4\%), and Rhodobacteraceae (1\%; Figure 5B). Most bacteria in the diet were Weissella and Streptococcus while the tank biofilm consisted of Rhodobacteraceae and Flavobacteriaceae.

Lactic acid bacteria from the Firmicutes phylum, particularly Streptococcus and Weissella, were found in higher abundance in the gut of fish fed low lipid and low n-3 diets as analyzed by LEfSe (Table 3 ). In contrast, fish fed high lipid diets had higher abundance of bacteria in three other phyla: Actinobacteria, Proteobacteria, and Verrucomicrobia.

\section{Functional Predictions of 16S rRNA Metagenome}

Overall, the predictive metagenomic pathways at KEGG Level 1 from the gut bacteria were dominated by metabolism and genetic information processing and to a lesser extent by environmental information processing and cellular processes (Figure 6A). Gut bacteria in fish fed high lipid diet with high n-3 showed increased $(p<0.001)$ functional pathways of genetic info processing while high lipid diet with low 


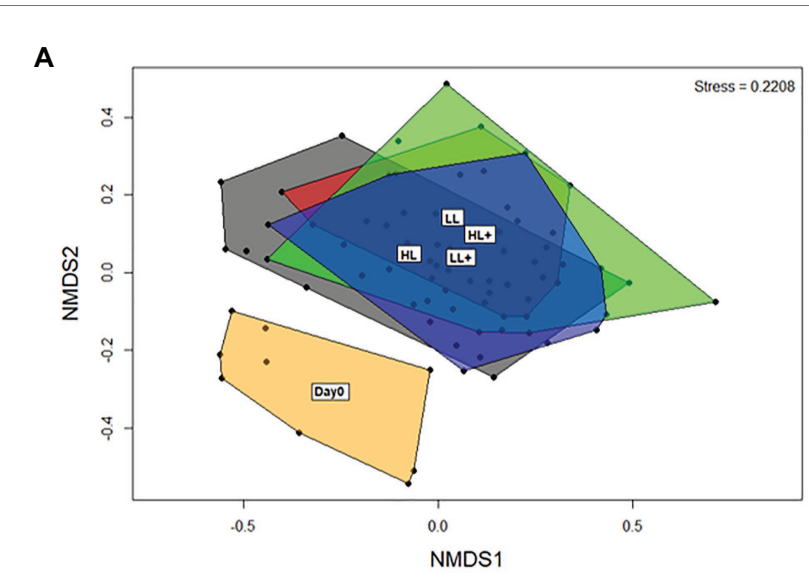

B

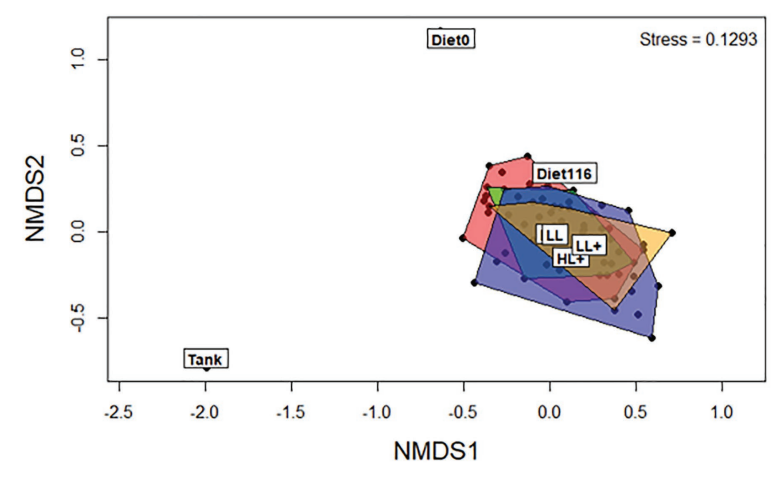

FIGURE 3 | Non-metric dimensional scaling (NMDS) plots of bacterial beta-diversity from the distal intestine of Atlantic salmon fed $\mathrm{HL}$ or $\mathrm{LL}$ diets with or without additional n-3 PUFA (+). Oxygen conditions were pooled together and fish were sampled on $(\mathbf{A})$ day $0(N=9)$ and 35 $(N=72)$, and (B) day $116(N=72)$. Intestine samples were compared to the commercial diet (Diet0), pooled experimental diets (Diet116), and tank biofilm (Tank; $N=3$ ).

n-3 showed activation of metabolic and cellular processing pathways. Within the metabolic pathway at KEGG Level 2, the metabolism of carbohydrates, amino acids, and nucleotides dominated followed to a lesser extent by energy, lipids, and vitamins (Figure 6B). The high lipid diet with high n-3 elevated $(p<0.001)$ metabolic pathways related to carbohydrates and nucleotides, whereas the high lipid diet with low n-3 elevated amino acid, vitamin, and lipid metabolic pathways. Within the lipid metabolic pathway at KEGG Level 3, the high lipid diet with $\mathrm{n}-3$ elevated $(p<0.05)$ metabolic pathways of glycerolipids, phospholipids, linoleic acid (18:2n-6), and arachidonic acid (20:4n-6; $p=0.096)$, whereas the high lipid (HL) diet elevated $(p<0.05)$ the biosynthesis of fatty acids, unsaturated fatty acids, lipid proteins, and steroids (Figure 6C). Alpha-linolenic acid $(18: 3 n-3)$ was elevated in fish fed the HL diet, but was not significant $(p=0.411)$. A higher abundance of Bifidobactericeae, Actinomyces, and Rhodococcus resulted in higher pathway activation of fatty acid synthase (Figure 7). There were no significant effects between high and low lipid or oxygen treatments (data not shown).

\section{DISCUSSION}

There has been a spike in the number of studies using highthroughput sequencing to characterize the gut microbes of salmonids including farmed species of Atlantic salmon (Zarkasi et al., 2014, 2016; Gajardo et al., 2016, 2017; Dehler et al., 2017; Rudi et al., 2018; Villasante et al., 2019), rainbow trout (Huyben et al., 2017, 2018, 2019; Lyons et al., 2017a,b,c; Michl et al., 2017; Bruce et al., 2018 ) and Arctic charr (Nyman et al., 2017). Of these, most dietary studies have investigated replacing fishmeal with vegetable, insect, or microbial protein sources. Similar to the present study, a study by Zarkasi et al. (2016) fed diets with high (300:400 g/kg) and low (200:500 g/ $\mathrm{kg}$ ) ratios of lipid:protein to Atlantic salmon post-smolts for 5-months and found that alpha-diversity of gut microbiota increased for fish fed high lipid diets, which decreased across all treatments over time. Declining alpha-diversity has been previously correlated with age as fish tend to have a reduced ability to filter microbial communities as they mature (Llewellyn et al., 2016; Burns et al., 2018; Heys et al., 2020). Two studies have examined the effect of replacing fish oil with vegetable oil on the gut microbiota of salmonids that drastically change the intake level of n-3 LC-PUFA, e.g., EPA and DHA (Ingerslev et al., 2014; Rudi et al., 2018). Compared to our results, Rudi et al. (2018) found similar alpha-diversity and betadiversity in the gut of Atlantic salmon smolts, especially the high abundance of Streptococcus, Weissella, Lactobacillus, Photobacterium, and Bradyrhizobium. However, the present study is unique in that it is the first to investigate the effect of both lipid:protein and levels of n-3 LC-PUFA as well as hypoxia on the microbiota of a salmonid species.

The effect of dietary n-3 PUFA on alpha-diversity (i.e., Shannon and Chao-1 indices) of gut microbes on day 116 (Table 2) has not been observed before in salmonid fishes. This was partially in agreement with our original hypothesis that alpha-diversity would decrease with higher n-3 LC-PUFA levels, which was only the case at the end of the trial. In humans, 45 year-old men that consumed 600 mg of n-3 PUFA every day over 2 weeks resulted in a reduced alpha-diversity of their gut microbes (Noriega et al., 2016). These authors attributed the decrease to a higher abundance of bacteria that produce short-chain fatty acids, such as Lactobacillus, although in the present study, we found a reduction of this genus and lower activation of fatty acid biosynthesis pathways in gut bacteria when fed high n-3 LC-PUFA diets (Figures 5, 6C). In gilthead seabream, feeding a low protein diet with high n-3 for 81 days had the lowest Shannon diversity of gut microbes sequenced from extracted bands from PCR-DGGE (Castro et al., 2019). In Atlantic salmon, Rudi et al. (2018) found that replacing fish oil with vegetable oil, low in n-3, had no effect on Shannon diversity of gut microbes in both freshwater and saltwater. In rainbow trout, Ingerslev et al. (2014) reported that replacing fish oil with rapeseed oil had no effect on Shannon diversity of gut microbes. However, both salmonid studies fed fish vegetable oil diets for only 20 and 23 days and the present study also confirmed that similarly there was no effect of n-3 on alpha-diversity on day 35 (Table 1). 

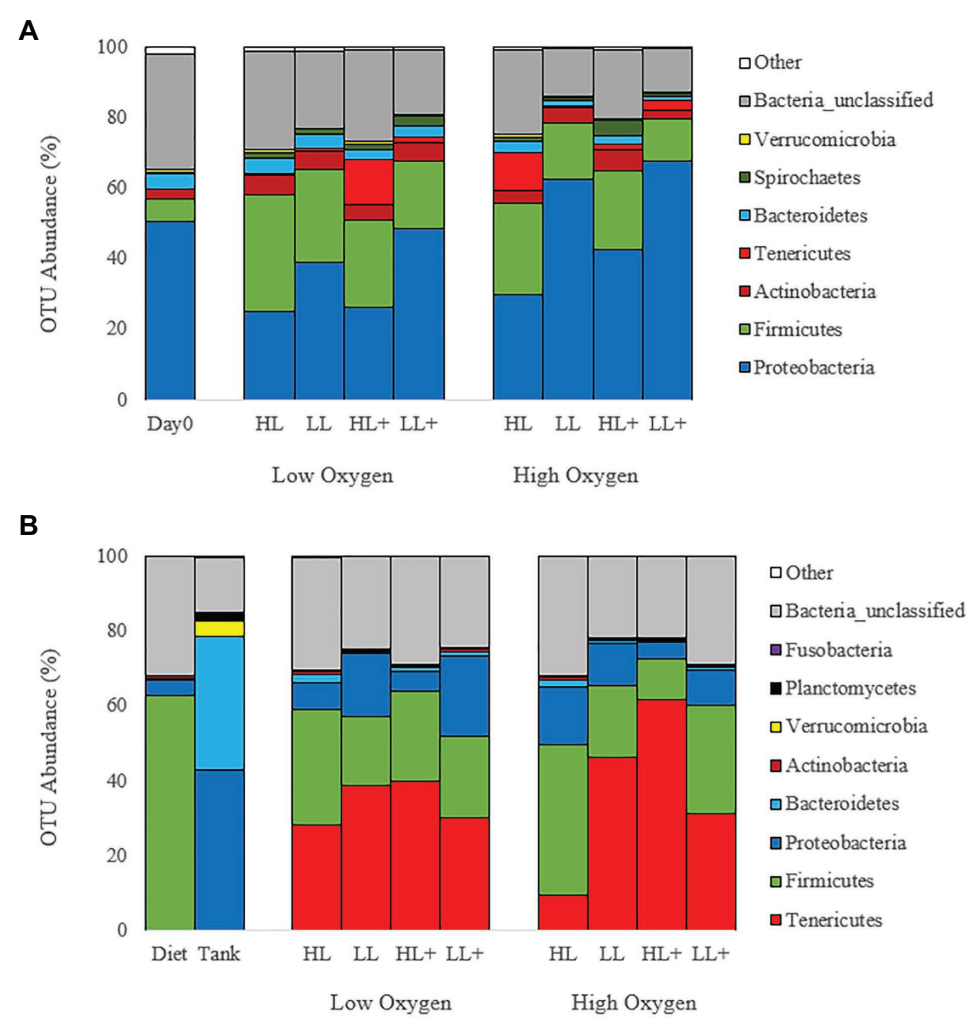

FIGURE 4 | Bar plots of bacteria (phyla level) from the distal intestine of Atlantic salmon fed HL or LL diets with or without additional n-3 PUFA (+) under high or low dissolved oxygen conditions sampled on $(\mathbf{A})$ day $0(N=9), 35(N=72)$ and $(\mathbf{B})$ day $116(N=72)$ along with diet and tank biofilm $(N=3)$.

In contrast, lipid level only influenced alpha-diversity in the short-term and was not evident at the end of the trial on day 116 (Table 2 and Figure 2). This agrees with a 5-month study by Zarkasi et al. (2016) that found alpha-diversity of gut bacteria was higher in Atlantic salmon fed a high lipid (low protein) diet compared to a control diet, although after some months, the diversities were equal (Zarkasi et al., 2016). One theory may be that alpha-diversity decreases over time and masks effect of large macronutrients, such as lipid, whereas n-3 influences metabolic pathways that require more time to alter gut microbial composition. On day 116 in the present study, Shannon diversity of gut microbiota were the highest in fish fed high lipid diets whereas diets with high n-3 suppressed diversity and indicated a lipid $\times$ n-3 interaction (Table 2). In a parallel study, Huyben et al. (2021) found that the digestibility of n-3 LC-PUFA was $99-100 \%$ compared to $95-98 \%$ of lipid for Atlantic salmon, which suggests that the amount of leftover lipid in the feces would influence the gut microbiota more than leftover n-3 and may have resulted in a delayed effect. Results from the present study indicate that alpha-diversity of gut microbes can be influenced in the short-term ( $<35$ days) by feeding different lipid levels while changes in $n-3$ levels only became evident after long-term feeding (36-116 days) and may have suppressed the beneficial effects of high lipid levels.

Changes in the beta-diversity (i.e., relative abundance) of gut bacteria due to changes in dietary n-3 PUFA level have been previously reported. Ingerslev et al. (2014) found that replacing fish oil with vegetable oil increased Firmicutes:Proteobacteria ratio, specifically genera of Streptococcus, Leuconostoc, and Weissella, which were also found in the present study (Table 3; Figures 4, 5). However, the diet also included high amounts of pea protein concentrated to replace fishmeal, thus the effects of the rapeseed oil on the gut microbiota are uncertain. In the present study, only a small volume of fish oil was replaced with olive oil in order to test the change in n-3 LC-PUFA rather than the oil source. Similar to our results, Zarkasi et al. (2017) found that Alivibrio, Vibrio, and Photobacterium were consistently dominant genera despite feeding different diets containing high and low lipid levels (200 and $300 \mathrm{~g} /$ $\mathrm{kg}$ ) to an in vitro salmon gut system. In vivo, Zarkasi et al. (2016) found that the high lipid:protein diet reduced the abundance of Proteobacteria, specifically Gammaproteobacteria (class level) and Alivibrio, in the gut of Atlantic salmon that was similar to the present study (Table 3 ). In the present study, genera of Streptococcus, Leuconostoc, and Weissella were the most abundant in the diet and had similar abundance in the gut at day 35 and 116, which indicates a direct diet-gut connection in terms of bacteria colonization (Figure 5). In contrast, OTUs of Rhodobacteraceae and Flavobacteriaceae from the tank biofilm were not present in the gut at a high abundance $(<0.1 \%)$. 
A

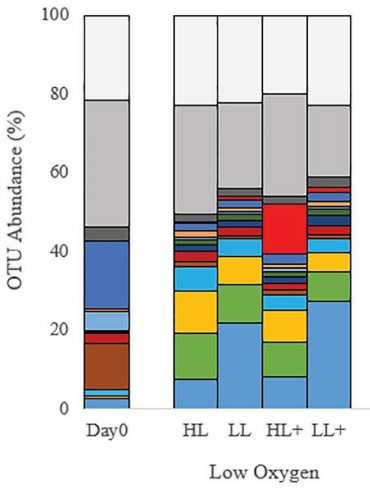

B
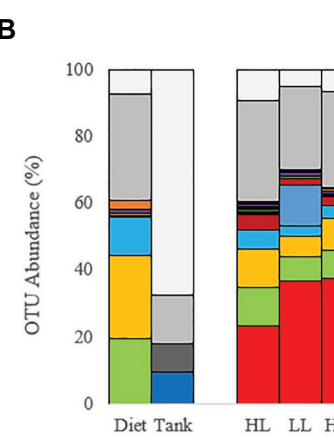

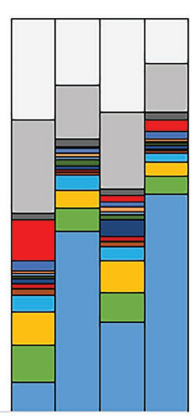

HL LL HL+ LL+

High Oxygen

口Other

$\square$ Bacteria unclassified

口Burkholderiales_unclassified

-Mycoplasmataceae_unclassified

$\square$ Rhodobacteraceae_unclassified

口Burkholderia

$\square$ Stenotrophomonas

aradyrhizobium

arevinema

aFlavobacterium

- Photobacterium

口Lactobacillus

口Weissella

$\square$ Streptococcus

$\square$ Aliivibrio

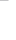

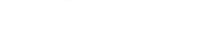

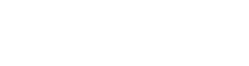
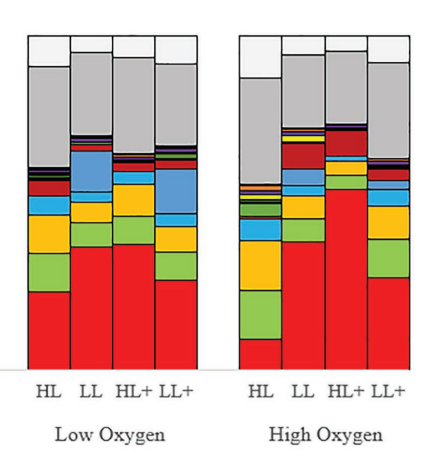
口Other
口Bacteria_unclassified
- Gammaproteobacteria_unclassified
口Lactobacillales_unclassified
-Burkholderiales_unclassified
$\square$ Rhodobacteraceae_unclassified
口Vibrio
घBradyrhizobium
$\square$ Photobacterium
口Moritella
- Mycoplasma
aAliivibrio
口Lactobacillus
口Weissella
Streptococcus
$\square$ Mycoplasmataceae_unclassified

FIGURE 5 | Bar plots of bacteria (genus level) from the distal intestine of Atlantic salmon fed HL or LL diets with or without additional n-3 PUFA (+) under high or low dissolved oxygen conditions sampled on $(\mathbf{A})$ day $0(N=9), 35(N=72)$ and $(\mathbf{B})$ day $116(N=72)$ along with diet and tank biofilm $(N=3)$.

TABLE 3 | Indicator operational taxonomic units (OTUs) of intestinal bacteria from Atlantic salmon ( $N=72)$ on day 116 that explain differences between dietary treatments identified by Linear discriminant analysis effect size (LEfSe).

\begin{tabular}{|c|c|c|c|c|c|}
\hline Phyla & Order & Genus & Diet & LDA & $p$-value \\
\hline Actinobacteria & Actinomycetales & Microbacteriaceae_unclassified & High Lipid & 2.0 & 0.033 \\
\hline \multirow[t]{5}{*}{ Firmicutes } & Lactobacillales & Lactobacillales_unclassified & Low Lipid \& Low n-3 & $2.1 / 2.3$ & $0.043 / 0.003$ \\
\hline & & Lactobacillus & Low n-3 & 2.3 & 0.035 \\
\hline & & Leuconostoc & Low n-3 & 3.0 & 0.033 \\
\hline & & Streptococcus & Low Lipid \& Low n-3 & $2.3 / 3.8$ & $<0.001 / 0.041$ \\
\hline & & Weissella & Low Lipid \& Low n-3 & $2.7 / 2.9$ & 0.034/0.012 \\
\hline \multirow[t]{5}{*}{ Proteobacteria } & Burkholderiales & Burkholderiales_unclassified & Low Lipid \& Low n-3 & $2.4 / 2.1$ & $<0.001 / 0.033$ \\
\hline & Enterobacteriales & Enterobacteriaceae_unclassified & High Lipid & 2.4 & 0.025 \\
\hline & Oceanospirillales & Chromohalobacter & High Lipid & 2.9 & 0.048 \\
\hline & Vibrionales & Aliivibrio & Low Lipid & 4.5 & $<0.001$ \\
\hline & & Photobacterium & Low n-3 & 2.8 & 0.021 \\
\hline Verrucomicrobia & Verrucomicrobiales & Verrucomicrobiaceae_unclassified & High Lipid & 2.6 & 0.030 \\
\hline
\end{tabular}

LDA; linear discriminant analysis.

Lactic acid bacteria have been identified as beneficial gut microbes; therefore, higher abundance in fish fed low lipid and low n-3 diets may indicate a more favorable bacterial community (Table 3; Figure 5). The genera of Streptococcus, Leuconostoc, and Weissella are lactic acid bacteria (Lactobacillales order) that are generally considered as favorable gut microbes due to their abilities to stimulate and enhance gut development, digestive function, mucosal tolerance, immune response, and disease resistance (Merrifield et al., 2010; Ringø et al., 2018).
Our results agree with an earlier study that showed feeding a marine diet with high PUFA (i.e., linoleic acid) depressed the viable counts of lactic acid bacteria, specifically Lactobacillus, in the gut of Arctic charr (Ringø, 1993). The intact lipopolysaccharide layer of Gram-negative microbes apparently screens the cells against medium and long-chain fatty acids and prevents their accumulation on the inner cell membrane, whereas Gram-positive microbes (e.g., lactic acid bacteria) cannot and become inhibited (Sheu and Freese, 1973). 
A

KEGG Level 1 -all pathways

$\square \mathrm{HL} \quad \mathrm{HL}+$

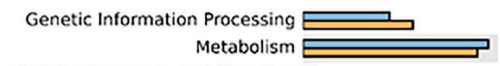

Cellular Processes and Signaling $P$

Cellular Processes p

Organismal Systems |

Poorly Characterized 日

Human Diseases 1

Environmental Information Processing

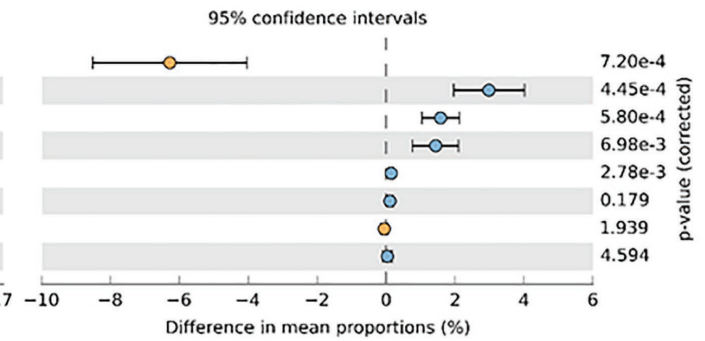

B

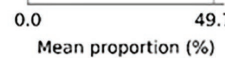

Difference in mean proportions (\%)

KEGG Level 2 -metabolic pathways

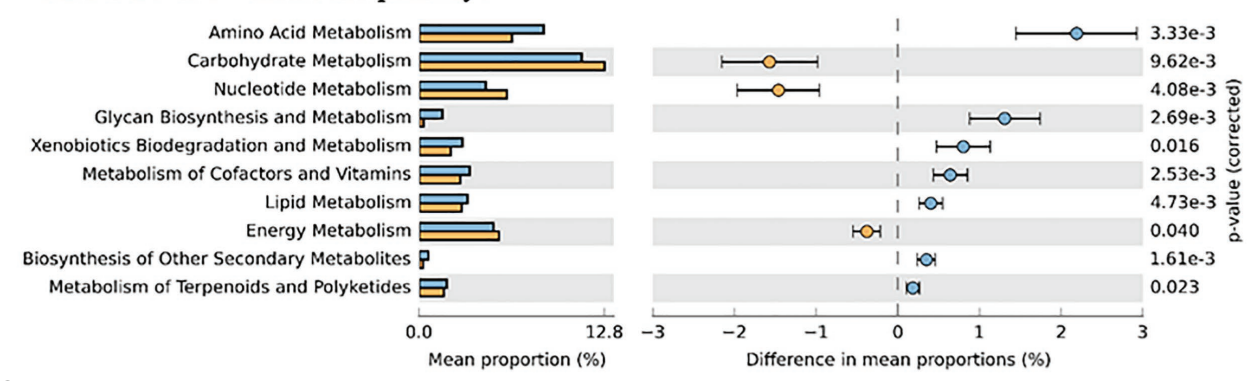

C

KEGG Level 3 - lipid metabolic pathways
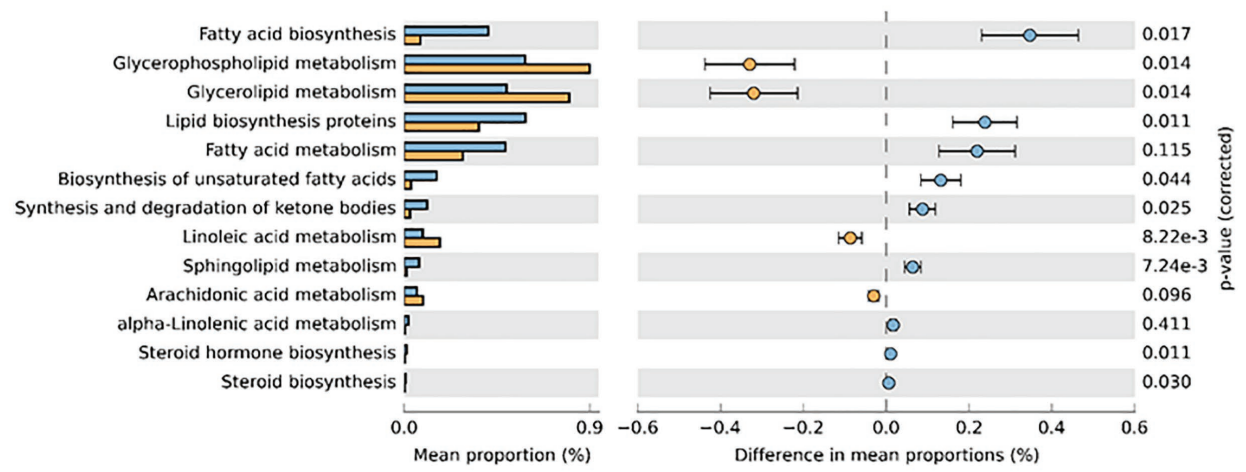

FIGURE 6 | Statistical Analysis of Taxonomic and Functional Profiles (STAMP) plots of predictive metagenomic analysis using phylogenetic investigation of communities by reconstruction of unobserved states (PICRUSt) to infer function of the distal intestine microbiome ( $N=72$ ) of Atlantic salmon on day 116 fed $\mathrm{HL}$ and high lipid with additional n-3 LC-PUFA (HL+). Kyoto Encyclopedia of Genes and Genomes (KEGG) Level 1 includes all pathways (A), two includes metabolic pathways (B), and three includes lipid metabolic pathways (C). $p$-values were generated by Welch's $t$-test with Bonferroni correction and $<0.05$ was considered significant.

Suppression of lactic acid bacteria by high inclusions of $\mathrm{n}-3$ LC-PUFA in the diet may reduce gut health; therefore, gut microbiota needs to be considered when optimizing n-3 levels for optimal growth performance of Atlantic salmon.

The increase in abundance of Tenericutes, specifically Mycoplasma (genus) and Mycoplasmataceae (order), in the fish gut from day 35 to 116 has been shown previously. Zarkasi et al. (2014) found higher abundance of Mycoplasma, Photobacterium, Alivibrio, and Vibrio after 13 months of rearing Atlantic salmon in Tasmanian sea-cages and increasing dietary lipid from approximately $230-330 \mathrm{~g} / \mathrm{kg}$. In contrast, abundance of Alivibrio was lower at the end of the trial and lower in fish fed high lipid diets in the present study (Table 3; Figure 5B). Other studies have found Tenericutes or Mycoplasma as a common inhabitant in the gut at high abundances of up to $83 \%$ in rainbow trout (Lyons et al., 2017c; Huyben et al., 2018) and 92\% in Atlantic salmon (Zarkasi et al., 2014; Llewellyn et al., 2016; Rudi et al., 2018). The most common species of Mycoplasma found in the present study was Mycoplasma penetrans, which has been highlighted as a candidate agent of transmissible tumors in the gut of zebrafish (Burns et al., 2018). These authors found that intestinal neoplasm was transmitted from afflicted to healthy fish through cohabitation while acquiring an increased abundance of Mycoplasma, indicating an infectious etiology rather than tumors caused by diet, water quality, or genetic background. Heys et al. (2020) found that Mycoplasma are not transient in the gut of Atlantic salmon but rather adapt 


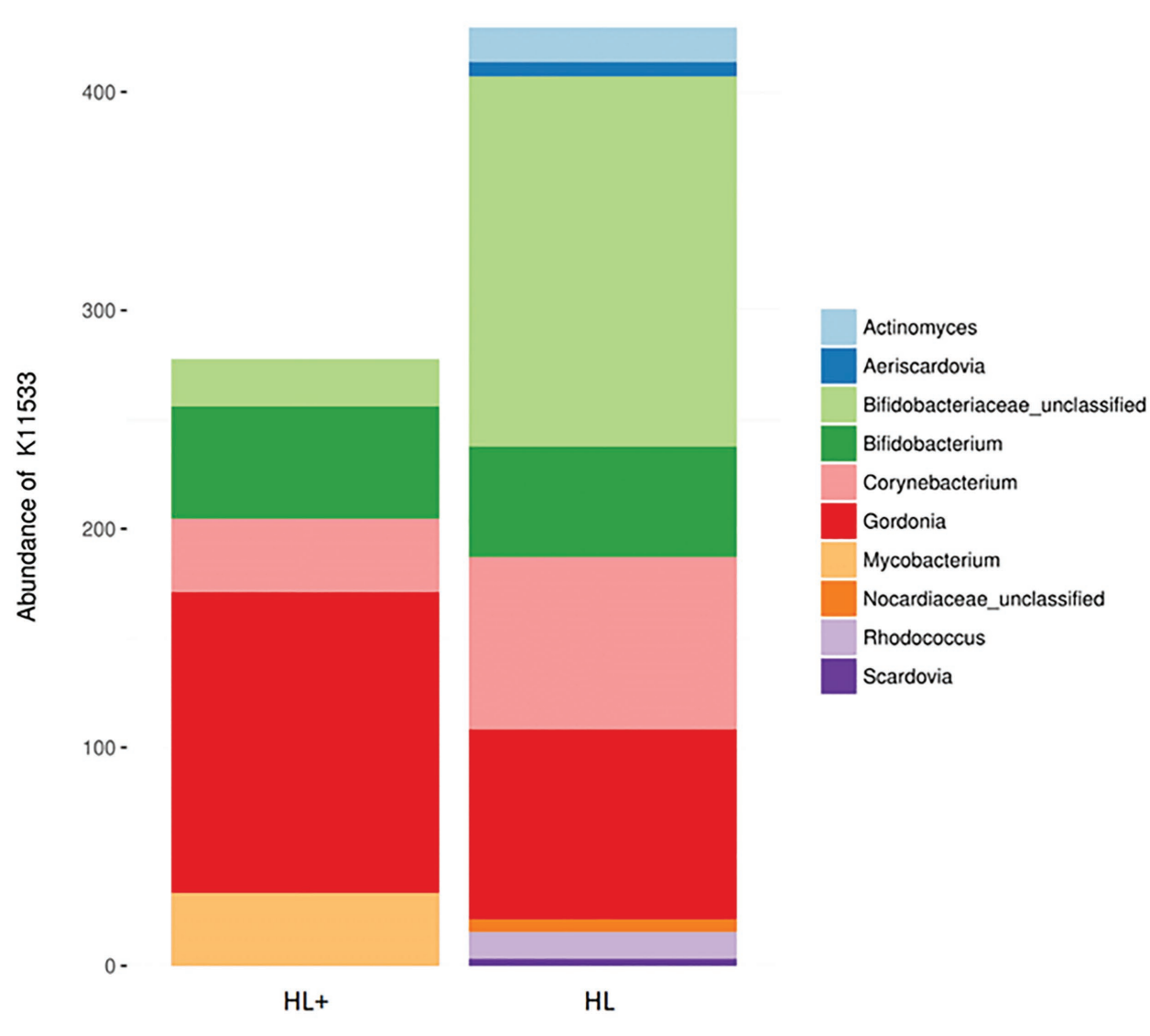

FIGURE 7 | Contribution of bacterial OTUs to the abundance of KEGG ortholog K11533 (fatty acid synthase - see Figure $6 C$ ) in the gut of Atlantic salmon ( $N=72$ ) fed high lipid with additional n-3 LC-PUFA (HL+) or HL.

to their host environment. In addition, both tumors and Mycoplasma have been found in the gut of older zebrafish and wild Atlantic salmon (Paquette et al., 2013; Llewellyn et al., 2016), as was the case in the present study as Mycoplasma was not abundant until day 116 (Figure 5). Despite the potential pathogenic nature of Mycoplasma, it can persist in healthy fish populations without clinical signs of disease (Burns et al., 2018), which was reflected in the present study since fish fed high lipid diet with high n-3 had the highest final weight and abundance of Mycoplasma and Mycoplasmataceae (Figure 5B). These fish also had reduced alpha-diversity in the gut, which has been associated with higher incidence of disease (Li et al., 2016; Parshukov et al., 2019). One theory may be that fish do not rely on the nutrient production from gut microbes as much if requirements are met by the diet consumed. In fish fed the high lipid diet with high $\mathrm{n}-3$, gut microbes had a higher activation of energy and carbohydrate metabolic pathways (Figure 6) that may represent a shift from specific nutrient production to energy acquisition. For instance, fish fed high lipid diets with low n-3 had higher metabolism of amino acids, vitamins, and lipids possibly in lieu of energy metabolism. More research is needed to determine the impacts of Mycoplasma, alpha-diversity, and predictive metagenomic function of gut microbes on the health and growth performance of fish.

Previous studies have found that the metabolism contributes the most to the predictive pathways of the salmonid gut microbiome, followed by information and cellular processing (Dehler et al., 2017; Lyons et al., 2017c; Yildirimer and Brown, 2018). Specifically, our results agree that a high proportion of pathways are attributed to amino acid, carbohydrate, and energy metabolism and to a lesser extent vitamin and lipid metabolism (Dehler et al., 2017; Lyons et al., 2017c). Very few studies exist that compare the impact of the gut microbiome on lipid metabolism in fish, despite a connection between n-3/n- 6 fatty acids and gut microbes. A study by Yildirimer and Brown (2018) found that a population of wild rainbow trout had lower lipid absorption and higher activation of lipid metabolism pathways, specifically linoleic acid (18:2n-6). Wild fine flounder (Paralichthys adspersus) were found to have higher activation of fatty acid metabolism and biosynthesis of unsaturated fatty acids pathways compared to farmed flounder presumably fed a formulated diet higher in lipid and LC-PUFA (Ramírez and Romero, 2017). These results agree with the current study where fish fed the high lipid diet with low n-3 LC-PUFA resulted in higher activation of lipid metabolism, specifically the biosynthesis 
of saturated and unsaturated fatty acids (Figures 6B,C). Other than glycerolipid metabolism, the only lipid metabolic pathway significantly affected by the high $\mathrm{n}-3$ diet was the metabolism of n-6 PUFAs linoleic acid (18:2n-6) and arachidonic acid (20:4n-6), which may be explained by higher levels in the diet. The pathway for fatty acid synthase was dominated by Actinobacteria, such as Bifidobacterium, Corynebacterium, and Gordonia (Figure 7), which have $<1 \%$ abundance in the gut but may be key contributors to lipid metabolism. In Arctic charr, feeding marine oil reduced viable counts of Corynebacterium compared to fish fed soybean and linseed oils (Ringø et al., 2002). In humans, consuming n-3 PUFA supplements increased abundance of Bifidobacterium (Watson et al., 2018), which produces short-chain fatty acids (i.e., butyrate) that can act as an energy source, improve cell signaling, and reduce inflammation in the gut (Noriega et al., 2016). These findings suggest that the gut microbiome can respond in efforts to compensate for a lack of $n-3$ in the diet by increasing the abundance of fattyacid producing bacteria. However, these results are only functional predictions based on existing reference genomes and deep shotgun sequencing of all genomes in each sample is the preferred technique (Langille et al., 2013).

We did not expect that a reduction in dissolved oxygen from 8.0 to $6.7 \mathrm{mg} / \mathrm{L}$ (93-78\% saturation) would have minor or no effects on viable plate counts, alpha-diversity, beta-diversity, indicator OTUs (LEfsE), or predictive metagenomics function of gut bacteria in Atlantic salmon. There was an initial effect of oxygen on viable plate counts and beta-diversity that may be due to reduced feed intake at the start of the experiment, while no effect of oxygen was found on day 116. In contrast, reduced oxygen from 6-7 to $3-4.5 \mathrm{mg} / \mathrm{L}$ resulted in lower alpha-diversity, different beta-diversity, altered metabolism pathways (e.g., fatty acid), and indicator OTUs of Chromatiales, Enterobacteriales, and Spirochaetales in the gut of four-eyed sleeper (Bostrychus sinensis; Fan et al., 2020). Reduced oxygen from 6.5 to $2.5 \mathrm{mg} / \mathrm{l}$ resulted in lower alpha-diversity, different beta-diversity, altered metabolism pathways (e.g., linoleic acid), and indicator OTUs of Clostridia and Lactobacillus in the gut of oriental river prawn (Macrobrachium nipponense; Sun et al., 2020). Reduced oxygen from 9.7 to $8.6 \mathrm{mg} / \mathrm{L}$ resulted in lower alpha-diversity and abundance of Lactobacillus in the gut of rainbow trout, although temperature was also a confounding factor in that study as it increased from 11 to $18^{\circ} \mathrm{C}$ (Huyben et al., 2018). Therefore, in the present study, the reduction in dissolved oxygen we believe was not severe enough to cause significant changes in gut microbiota of Atlantic salmon.

\section{CONCLUSION}

This study investigated the effects of two dietary components, lipid, and n-3 LC-PUFA level, as well as a common chronic stressor, hypoxia, on the gut microbiome of Atlantic salmon using agar plating, 16S rRNA gene next-generation sequencing, and bioinformatic and predictive metagenomic analyses. This is the first study to find significant effects of both dietary n-3 and a lipid $\times \mathrm{n}-3$ interaction on the alpha and beta-diversities of gut bacteria in salmonids. There were stark differences in the effects of dietary lipid and n-3 levels on gut microbes between days 0,35 , and 116. Initially, high lipid diets (low protein) increased alpha-diversity and by the end of the trial high n-3 levels reduced alpha-diversity, possibly suppressing beneficial effects of high lipid. There was a general reduction of alphadiversity over time. Between diets, beta-diversity and phyla abundance were similar, except for some genera, such as lactic acid bacteria (e.g., Streptococcus, Weissella, and Lactobacillus), associated with fish fed low lipid (high protein) and low n-3 diets. Between time points, the relative abundance of the Tenericutes phylum (i.e., Mycoplasma) increased 10-fold to approximately $36 \%$ of gut microbes, which supports previous findings that older fish are less able to filter pathogenic gut bacteria. At high oxygen, fish fed the high lipid diet with high $\mathrm{n}-3$ (closest to a commercial analogous diet) had reduced alpha-diversity, lowest abundance of lactic acid bacteria (11\%), and highest abundance of Mycoplasma/Mycoplasmataceae (62\%). The only effect of oxygen was on the viable counts of bacteria and an initial effect on beta-diversity; otherwise, the reduction in dissolved oxygen from 8.0 to $6.7 \mathrm{mg} / \mathrm{l}$ may have been too minor to influence the alpha and beta-diversities or predictive functions of gut microbes at the end of the trial. These findings suggest these fish may have a poor gut microbiota or dysbiosis even though this group had the largest final fish weight, thus more research needs to determine the function of Mycoplasma and relation to fish growth performance. With regard to predictive function, PICRUSt analysis showed that feeding lower levels of n-3 resulted in higher metabolism of saturated and unsaturated fatty acids by the gut microbiome, potentially as an effort to compensate for low n-3 in the diet. In summary, our results indicate that the viable plate counts, alpha-diversity, beta-diversity, and predictive function of gut bacteria in Atlantic salmon post-smolts are influenced by dietary lipid:protein ratio and n-3 LC-PUFA levels over time, with only minor effects of dissolved oxygen.

\section{DATA AVAILABILITY STATEMENT}

The original contributions presented in the study are publicly available. This data can be found here: https://www.ncbi.nlm. nih.gov/bioproject/PRJNA650141.

\section{ETHICS STATEMENT}

The animal study was reviewed and approved by Animal Welfare and Ethical Review Body (reference AWERB/1617/84), UK Home Office.

\section{AUTHOR CONTRIBUTIONS}

The experiment was planned by BG, DH, and BRu. The fish were measured and sampled for intestinal content by DH, BG, and BRo. The lab work and sequencing on the 16S rRNA diversity was done by $\mathrm{DH}$ and BRo. Bioinformatics, statistics, 
and metagenomic analysis were done by $\mathrm{DH}$ and $\mathrm{MB}$. The manuscript was written by $\mathrm{DH}$ and all co-authors contributed revisions and approved the final version.

\section{FUNDING}

Funding for this study was provided by the Norwegian Research Council (HAVBRUK2 project ES576272), Norwegian Seafood Research Fund (FHF), and the University of Stirling (UoS).

\section{REFERENCES}

Asaduzzaman, M., Iehata, S., Akter, S., Kader, M. A., Ghosh, S. K., Khan, M. N. A., et al. (2018). Effects of host gut-derived probiotic bacteria on gut morphology, microbiota composition and volatile short chain fatty acids production of Malaysian Mahseer Tor tambroides. Aquac. Rep. 9, 53-61. doi: 10.1016/j. aqrep.2017.12.003

Bou, M., Berge, G. M., Baeverfjord, G., Sigholt, T., Østbye, T. -K., Romarheim, O. H., et al. (2017). Requirements of n-3 very long-chain PUFA in Atlantic salmon (Salmo salar L): effects of different dietary levels of EPA and DHA on fish performance and tissue composition and integrity. Br. J. Nutr. 117, 30-47. doi: $10.1017 /$ S0007114516004396

Bruce, T. J., Neiger, R. D., and Brown, M. L. (2018). Gut histology, immunology and the intestinal microbiota of rainbow trout, Oncorhynchus mykiss (Walbaum), fed process variants of soybean meal. Aquac. Res. 49, 492-504. doi: 10.1111/ are. 13480

Burns, A. R., Watral, V., Sichel, S., Spagnoli, S., Banse, A. V., Mittge, E., et al. (2018). Transmission of a common intestinal neoplasm in zebrafish by cohabitation. J. Fish Dis. 41, 569-579. doi: 10.1111/jfd.12743

Caporaso, J. G., Lauber, C. L., Walters, W. A., Berg-Lyons, D., Lozupone, C. A., Turnbaugh, P. J., et al. (2011). Global patterns of $16 \mathrm{~S}$ rRNA diversity at a depth of millions of sequences per sample. Proc. Natl. Acad. Sci. U. S. A. 108, 4516-4522. doi: 10.1073/pnas.1000080107

Castro, C., Couto, A., Diógenes, A. F., Corraze, G., Panserat, S., Serra, C. R., et al. (2019). Vegetable oil and carbohydrate-rich diets marginally affected intestine histomorphology, digestive enzymes activities, and gut microbiota of gilthead sea bream juveniles. Fish Physiol. Biochem. 45, 681-695. doi: 10.1007/s10695-018-0579-9

Cole, J. R., Wang, Q., Fish, J. A., Chai, B., McGarrell, D. M., Sun, Y., et al. (2013). Ribosomal database project: data and tools for high throughput rRNA analysis. Nucleic Acids Res. 42, D633-D642. doi: 10.1093/nar/gkt1244

Dehler, C. E., Secombes, C. J., and Martin, S. A. (2017). Environmental and physiological factors shape the gut microbiota of Atlantic salmon parr (Salmo salar L.). Aquaculture 467, 149-157. doi: 10.1016/j.aquaculture.2016.07.017

Desantis, T. Z., Hugenholtz, P., Larsen, N., Rojas, M., Brodie, E. L., Keller, K., et al. (2006). Greengenes, a chimera-checked 16S rRNA gene database and workbench compatible with ARB. Appl. Environ. Microbiol. 72, 5069-5072. doi: 10.1128/AEM.03006-05

Fan, S., Li, H., and Zhao, R. (2020). Effects of normoxic and hypoxic conditions on the immune response and gut microbiota of Bostrichthys sinensis. Aquaculture 525:735336. doi: 10.1016/j.aquaculture.2020.735336

Gajardo, K., Jaramillo-Torres, A., Kortner, T. M., Merrifield, D. L., Tinsley, J., Bakke, A. M., et al. (2017). Alternative protein sources in the diet modulate microbiota and functionality in the distal intestine of Atlantic salmon (Salmo salar). Appl. Environ. Microbiol. 83:e02615. doi: 10.1128/AEM.02615-16

Gajardo, K., Rodiles, A., Kortner, T. M., Krogdahl, Å., Bakke, A. M., Merrifield, D. L., et al. (2016). A high-resolution map of the gut microbiota in Atlantic salmon (Salmo salar): a basis for comparative gut microbial research. Sci. Rep. 6:30893. doi: 10.1038/srep30893

Glencross, B. D., Tocher, D. R., Matthew, C., and Bell, J. G. (2014). Interactions between dietary docosahexaenoic acid and other long-chain polyunsaturated fatty acids on performance and fatty acid retention in post-smolt Atlantic salmon (Salmo salar). Fish Physiol. Biochem. 40, 1213-1227. doi: 10.1007/ s10695-014-9917-8

\section{ACKNOWLEDGMENTS}

We are especially grateful to staff at the Marine Environmental Research Lab in Machrihanish, UK and the Institute of Aquaculture, Stirling, UK. In particular, special thanks to Chessor Matthew, Anna Krzyskow, Jessica Di Toro, Teresa Grobler, Xu Gong, Pedro Munoz, and Tarah Mayes for their assistance in raising and sampling fish, as well as Rona Werner, Stuart McMillan, Chris Payne, and John Taggart for their assistance in the bacteriology and molecular labs.

Heys, C., Cheaib, B., Busetti, A., Kazlauskaite, R., Maier, L., Sloan, W. T., et al. (2020). Neutral processes dominate microbial community assembly in Atlantic salmon, Salmo salar. Appl. Environ. Microbiol. 86, e02283-e02302. doi: 10.1128/AEM.02283-19

Huyben, D., Grobler, T., Matthew, C., Bou, M., Ruyter, B., and Glencross, B. (2021). Requirement for omega-3 long-chain polyunsaturated fatty acids by Atlantic salmon is relative to the dietary lipid level. Aquaculture 531:735805. doi: $10.1016 /$ j.aquaculture.2020.735805

Huyben, D., Nyman, A., Vidaković, A., Passoth, V., Moccia, R., Kiessling, A., et al. (2017). Effects of dietary inclusion of the yeasts Saccharomyces cerevisiae and Wickerhamomyces anomalus on gut microbiota of rainbow trout. Aquaculture 473, 528-537. doi: 10.1016/j.aquaculture.2017.03.024

Huyben, D., Sun, L., Moccia, R., Kiessling, A., Dicksved, J., and Lundh, T. (2018). Dietary live yeast and increased water temperature influence the gut microbiota of rainbow trout. J. Appl. Microbiol. 124, 1377-1392. doi: 10.1111/jam. 13738

Huyben, D., Vidaković, A., Werner Hallgren, S., and Langeland, M. (2019). High-throughput sequencing of gut microbiota in rainbow trout (Oncorhynchus mykiss) fed larval and pre-pupae stages of black soldier fly (Hermetia illucens). Aquaculture 500, 485-491. doi: 10.1016/j.aquaculture.2018.10.034

Ingerslev, H. C., von Gersdorff Jørgensen, L., Lenz Strube, M., Larsen, N., Dalsgaard, I., Boye, M., et al. (2014). The development of the gut microbiota in rainbow trout (Oncorhynchus mykiss) is affected by first feeding and diet type. Aquaculture 424-425, 24-34. doi: 10.1016/j.aquaculture.2013.12.032

Kanehisa, M., and Goto, S. (2000). KEGG: Kyoto encyclopedia of genes and genomes. Nucleic Acids Res. 28, 27-30. doi: 10.1093/nar/28.1.27

Kihara, M., and Sakata, T. (1997). Fermentation of dietary carbohydrates to short-chain fatty acids by gut microbes and its influence on intestinal morphology of a detritivorous teleost tilapia (Oreochromis niloticus). Comp. Biochem. Physiol. A Physiol. 118, 1201-1207. doi: 10.1016/S03009629(97)00052-2

Kozich, J. J., Westcott, S. L., Baxter, N. T., Highlander, S. K., and Schloss, P. D. (2013). Development of a dual-index sequencing strategy and curation pipeline for analyzing amplicon sequence data on the MiSeq Illumina sequencing platform. Appl. Environ. Microbiol. 79, 5112-5120. doi: 10.1128/ AEM.01043-13

Langille, M. G. I., Zaneveld, J., Caporaso, J. G., McDonald, D., Knights, D., Reyes, J. A., et al. (2013). Predictive functional profiling of microbial communities using 16S rRNA marker gene sequences. Nat. Biotechnol. 31, 814-821. doi: 10.1038/nbt.2676

Li, T., Long, M., Ji, C., Shen, Z., Gatesoupe, F. -J., Zhang, X., et al. (2016). Alterations of the gut microbiome of largemouth bronze gudgeon (Coreius guichenoti) suffering from furunculosis. Sci. Rep. 6:30606. doi: 10.1038/ srep30606

Llewellyn, M. S., McGinnity, P., Dionne, M., Letourneau, J., Thonier, F., Carvalho, G. R., et al. (2016). The biogeography of the Atlantic salmon (Salmo salar) gut microbiome. ISME J. 10, 1280-1284. doi: 10.1038/ ismej.2015.189

Lyons, P. P., Turnbull, J. F., Dawson, K. A., and Crumlish, M. (2017a). Effects of low-level dietary microalgae supplementation on the distal intestinal microbiome of farmed rainbow trout Oncorhynchus mykiss (Walbaum). Aquac. Res. 48, 2438-2452. doi: 10.1111/are.13080

Lyons, P. P., Turnbull, J. F., Dawson, K. A., and Crumlish, M. (2017b). Exploring the microbial diversity of the distal intestinal lumen and mucosa of farmed 
rainbow trout Oncorhynchus mykiss (Walbaum) using next generation sequencing (NGS). Aquac. Res. 48, 77-91. doi: 10.1111/are.12863

Lyons, P. P., Turnbull, J. F., Dawson, K. A., and Crumlish, M. (2017c). Phylogenetic and functional characterization of the distal intestinal microbiome of rainbow trout Oncorhynchus mykiss from both farm and aquarium settings. J. Appl. Microbiol. 122, 347-363. doi: 10.1111/jam.13347

Merrifield, D. L., Dimitroglou, A., Foey, A., Davies, S. J., Baker, R. T. M., Bøgwald, J., et al. (2010). The current status and future focus of probiotic and prebiotic applications for salmonids. Aquaculture 302, 1-18. doi: 10.1016/j. aquaculture.2010.02.007

Michl, S. C., Ratten, J. -M., Beyer, M., Hasler, M., Laroche, J., and Schulz, C. (2017). The malleable gut microbiome of juvenile rainbow trout (Oncorhynchus mykiss): diet-dependent shifts of bacterial community structures. PLoS One 12:e0177735. doi: 10.1371/journal.pone.0177735

Noriega, B. S., Sanchez-Gonzalez, M. A., Salyakina, D., and Coffman, J. (2016). Understanding the impact of omega-3 rich diet on the gut microbiota. Case Rep. Med. 2016:3089303. doi: 10.1155/2016/3089303

Nyman, A., Huyben, D., Lundh, T., and Dicksved, J. (2017). Effects of microbeand mussel-based diets on the gut microbiota in Arctic charr (Salvelinus alpinus). Aquac. Rep. 5, 34-40. doi: 10.1016/j.aqrep.2016.12.003

Oksanen, J., Blanchet, F., Friendly, M., Kindt, R., Legendre, P., McGlinn, D., et al. (2018). Vegan: community ecology package. R package version 2.5-1. Available at: https://CRAN.R-project.org/package=vegan (Accessed April 22, 2020).

Paquette, C. E., Kent, M. L., Buchner, C., Tanguay, R. L., Guillemin, K., Mason, T. J., et al. (2013). A retrospective study of the prevalence and classification of intestinal neoplasia in zebrafish (Danio rerio). Zebrafish 10, 228-236. doi: 10.1089/zeb.2012.0828

Parks, D. H., Tyson, G. W., Hugenholtz, P., and Beiko, R. G. (2014). STAMP: statistical analysis of taxonomic and functional profiles. Bioinformatics 30, 3123-3124. doi: 10.1093/bioinformatics/btu494

Parshukov, A. N., Kashinskaya, E. N., Simonov, E. P., Hlunov, O. V., Izvekova, G. I., Andree, K. B., et al. (2019). Variations of the intestinal gut microbiota of farmed rainbow trout, Oncorhynchus mykiss (Walbaum), depending on the infection status of the fish. J. Appl. Microbiol. 127, 379-395. doi: 10.1111/ jam. 14302

Pinheiro, J., Bates, D., Debroy, S., and Sarkar, D. (2014). R Package nlme: linear and nonlinear mixed effects models. $\mathrm{R}$ package version 3.1-117. Available at: http://CRAN.R-project.org/package=nlme (Accessed April 22, 2020).

Quast, C., Pruesse, E., Yilmaz, P., Gerken, J., Schweer, T., Yarza, P., et al. (2012). The SILVA ribosomal RNA gene database project: improved data processing and web-based tools. Nucleic Acids Res. 41, D590-D596. doi: 10.1093/nar/gks1219

Ramírez, C., and Romero, J. (2017). Fine flounder (Paralichthys adspersus) microbiome showed important differences between wild and reared specimens. Front. Microbiol. 8:271. doi: 10.3389/fmicb.2017.00271

R-Core-Team (2015). "R: A language and environment for statistical computing." 3.2.2 Edn. Vienna, Austria: R Foundation for Statistical Computing.

Ringø, E. (1993). Does dietary linoleic acid affect intestinal microflora in Arctic charr. Salvelinus alpinus (L.)? Aquac. Res. 24, 133-135. doi: 10.1111/j.13652109.1993.tb00837.x

Ringø, E., Hossein, S., Ghosh, K., Doan, H. V., Beck, B. R., and Song, S. (2018). Lactic acid bacteria in finfish-an update. Front. Microbiol. 9:1818. doi: $10.3389 /$ fmicb.2018.01818

Ringø, E., Lødemel, J. B., Myklebust, R., Jensen, L., Lund, V., Mayhew, T. M., et al. (2002). The effects of soybean, linseed and marine oils on aerobic gut microbiota of Arctic charr Salvelinus alpinus L. before and after challenge with Aeromonas salmonicida ssp. salmonicida. Aquac. Res. 33, 591-606. doi: 10.1046/j.1365-2109.2002.00697.x

Rognes, T., Flouri, T., Nichols, B., Quince, C., and Mahé, F. (2016). VSEARCH: a versatile open source tool for metagenomics. PeerJ 4:e2584. doi: 10.7717/peerj.2584

Rudi, K., Angell, I. L., Pope, P. B., Vik, J. O., Sandve, S. R., and Snipen, L. -G. (2018). Stable core gut microbiota across the freshwater-to-saltwater transition for farmed Atlantic salmon. Appl. Environ. Microbiol. 84, e01974-e01991. doi: 10.1128/AEM.01974-17

Ruyter, B., Røsjø, C., Einen, O., and Thomassen, M. S. (2000). Essential fatty acids in Atlantic salmon: effects of increasing dietary doses of n-6 and n-3 fatty acids on growth, survival and fatty acid composition of liver, blood and carcass. Aquac. Nutr. 6, 119-127. doi: 10.1046/j.1365-2095.2000.00137.x

Salter, S. J., Cox, M. J., Turek, E. M., Calus, S. T., Cookson, W. O., Moffatt, M. F., et al. (2014). Reagent and laboratory contamination can critically impact sequence-based microbiome analyses. BMC Biol. 12:87. doi: 10.1186/ s12915-014-0087-z

Schloss, P. D., Westcott, S. L., Ryabin, T., Hall, J. R., Hartmann, M., Hollister, E. B., et al. (2009). Introducing mothur: open-source, platform-independent, community-supported software for describing and comparing microbial communities. Appl. Environ. Microbiol. 75, 7537-7541. doi: 10.1128/ AEM.01541-09

Segata, N., Izard, J., Waldron, L., Gevers, D., Miropolsky, L., Garrett, W. S., et al. (2011). Metagenomic biomarker discovery and explanation. Genome Biol. 12:R60. doi: 10.1186/gb-2011-12-6-r60

Semova, I., Carten, J. D., Stombaugh, J., Mackey, L. C., Knight, R., Farber, S. A., et al. (2012). Microbiota regulate intestinal absorption and metabolism of fatty acids in the zebrafish. Cell Host Microbe 12, 277-288. doi: 10.1016/j. chom.2012.08.003

Sheu, C. W., and Freese, E. (1973). Lipopolysaccharide layer protection of gram-negative bacteria against inhibition by long-chain fatty acids. J. Bacteriol. 115, 869-875. doi: 10.1128/JB.115.3.869-875.1973

Sun, S., Yang, M., Fu, H., Ge, X., and Zou, J. (2020). Altered intestinal microbiota induced by chronic hypoxia drives the effects on lipid metabolism and the immune response of oriental river prawn Macrobrachium nipponense. Aquaculture 526:735431. doi: 10.1016/j.aquaculture.2020.735431

Villasante, A., Ramírez, C., Catalán, N., Opazo, R., Dantagnan, P., and Romero, J. (2019). Effect of dietary carbohydrate-to-protein ratio on gut microbiota in Atlantic salmon (Salmo salar). Animals 9:89. doi: 10.3390/ani9030089

Watson, H., Mitra, S., Croden, F. C., Taylor, M., Wood, H. M., Perry, S. L., et al. (2018). A randomised trial of the effect of omega-3 polyunsaturated fatty acid supplements on the human intestinal microbiota. Gut 67, 1974-1983. doi: 10.1136/gutjnl-2017-314968

Yildirimer, C. C., and Brown, K. H. (2018). Intestinal microbiota lipid metabolism varies across rainbow trout (Oncorhynchus mykiss) phylogeographic divide. J. Appl. Microbiol. 125, 1614-1625. doi: 10.1111/jam.14059

Zarkasi, K. Z., Abell, G. C., Taylor, R. S., Neuman, C., Hatje, E., Tamplin, M. L., et al. (2014). Pyrosequencing based characterization of gastrointestinal bacteria of Atlantic salmon (Salmo salar L.) within a commercial mariculture system. J. Appl. Microbiol. 117, 18-27. doi: 10.1111/jam.12514

Zarkasi, K. Z., Taylor, R. S., Abell, G. C. J., Tamplin, M. L., Glencross, B. D., and Bowman, J. P. (2016). Atlantic salmon (Salmo salar L.) gastrointestinal microbial community dynamics in relation to digesta properties and diet. Microb. Ecol. 71, 589-603. doi: 10.1007/s00248-015-0728-y

Zarkasi, K. Z., Taylor, R. S., Glencross, B. D., Abell, G. C., Tamplin, M. L., and Bowman, J. P. (2017). In vitro characteristics of an Atlantic salmon (Salmo salar L.) hind gut microbial community in relation to different dietary treatments. Res. Microbiol. 168, 751-759. doi: 10.1016/j. resmic.2017.07.003

Conflict of Interest: The authors declare that the research was conducted in the absence of any commercial or financial relationships that could be construed as a potential conflict of interest.

Copyright (C) 2020 Huyben, Roehe, Bekaert, Ruyter and Glencross. This is an open-access article distributed under the terms of the Creative Commons Attribution License (CC BY). The use, distribution or reproduction in other forums is permitted, provided the original author(s) and the copyright owner(s) are credited and that the original publication in this journal is cited, in accordance with accepted academic practice. No use, distribution or reproduction is permitted which does not comply with these terms. 